\title{
Solution of a dual integral equation arising in the contact problems of elasticity theory with the full Fourier series as the right-hand side
}

\author{
Andrey Vasiliev ${ }^{1,2 *}$, Sergei Volkov ${ }^{1,2}$ \\ ${ }^{1}$ Research Institute of Mechanics, National Research Lobachevsky State University of Nizhni Novgorod, 603950, Nizhni Novgorod, \\ Russia \\ ${ }^{2}$ Research and Education Center "Materials", Don State Technical University, 344000, Rostov-on-Don, Russia
}

\begin{abstract}
A class of dual integral equations is analyzed which arises in solution of a wide range of plane and antiplane contact problems of elasticity theory for a half-plane with functionally graded coating. In particular, a similar equation arises in solution of the contact problem on indentation in the presence of tangential stresses on a surface. The solution of the dual integral equation is sought in the form of a sum of even and odd functions. It makes possible to reduce the problem to independent solution of two dual integral equations over odd and even functions. Kernel transform of these equations is approximated by a product of fractional quadratic functions. The solution of dual integral equations is constructed in approximated analytical form by the bilateral asymptotic method. The expressions obtained are asymptotically exact for small and large values of a characteristic geometrical parameter.
\end{abstract}

\section{Introduction}

Contact mechanics attracts the researchers from all over the world for more than a century. For solution of contact problems, as well as the other mixed boundary value problems, methods based on complex analysis, the theory of potential functions and the theory of dual and singular integral equations are widely used [Ошибка! Источник ссылки не найден., 1].

From the last decade of the previous century functionally graded (continuously inhomogeneous) and layered coatings are actively studied [3-28]. Deformation of such materials is investigated caused by only mechanical loading [3-7], simultaneous mechanical and thermal loading [8-10], electromechanical loading $[11,12]$, etc. Other important research directions are fracture of such materials [13], deformation of stiff structures (beams and plates) made from such materials $[14,15]$ as well as bending of beams and plates lying on such materials [16, 17], etc. Functionally graded and layered composite coatings are used for protection from wear, erosion, oxidation forced by high temperature, cracks formations, etc.

Contact problems for functionally graded or layered coatings are usually reduced to the solution of singular integral equations or dual integral equations, which requires development of special methods. Among methods of solution these equations the following should be noticed: regular [18] and singular [19] asymptotic methods, orthogonal polynomials [20], collocation [21] and bilateral asymptotic [22] methods. The advantages of the bilateral asymptotic method in comparison with the others consist in following: the solution is constructed in the approximated analytical form and has high accuracy for coatings of any thickness; the method is effective for arbitrary variation of properties in depth of the coating.

The present paper addresses the solution of one class of dual integral equations arising in the solution of wide range of plane and antiplane contact problems, see for example $[5,17,24,25]$. Feature of the paper is that the right-hand side of the integral equation is represented by the full Fourier series that can be used to model nonsymmetric contact. The solution of this dual integral equation is of interest for the tribology, in particular, in modeling of lubricated contact of solids with functionally graded or layered coatings [25-28].

\section{Solution of dual integral equation}

Let us consider the dual integral equation:

$$
\begin{aligned}
& \int_{-\infty}^{\infty} Q(\alpha)|\alpha|^{-1} L(\alpha \lambda) e^{-i x \alpha} d \alpha=2 \pi f(x),|x| \leq 1 \\
& \int_{-\infty}^{\infty} Q(\alpha) e^{-i x \alpha} d \alpha=0,|x|>1
\end{aligned}
$$

Here $Q(\alpha)$ is the Fourier image of function $q(x)$ to be obtained, $\lambda$ is real parameter (usually, describing geometrical properties), $L(\alpha \lambda)$ is the kernel transform of integral equation possessing:

* Corresponding author: andre.vasiliev@gmail.com 


$$
\begin{aligned}
& L(\alpha)=A+B|\alpha|+C \alpha^{2}+O\left(|\alpha|^{3}\right), \quad \alpha \rightarrow 0 \\
& L(\alpha)=1+D|\alpha|^{-1}+E \alpha^{-2}+O\left(|\alpha|^{-3}\right), \quad \alpha \rightarrow \infty
\end{aligned}
$$

Function $f(x)$ can be represented by the full Fourier series:

$$
f(x)=a_{0}+\sum_{n=1}^{\infty}\left(a_{n} \cos (n \pi x)+b_{n} \sin (n \pi x)\right)
$$

Let us represent $q(x)$ in the form of sum of even $q_{+}(x)$ and odd $q_{-}(x)$ functions. Then for Fourier images the following equation is satisfied:

$$
Q(\alpha)=Q_{+}(\alpha)+i Q_{-}(\alpha)
$$

Substitution of (2) into (1) makes it possible to split the dual integral equation into two independent dual integral equations:

$$
\begin{aligned}
& \int_{-\infty}^{\infty} \frac{Q_{-}(\alpha)}{|\alpha|} L(\alpha \lambda) \sin (\alpha x) d \alpha= \\
& =-2 \pi \sum_{n=1}^{\infty} b_{n} \sin (n \pi x),|x| \leq 1 \\
& \int_{-\infty}^{\infty} Q_{-}(\alpha) \sin (\alpha x) d \alpha=0,|x|>1 \\
& \int_{-\infty}^{\infty} \frac{Q_{+}(\alpha)}{|\alpha|} L(\alpha \lambda) \cos (\alpha x) d \alpha= \\
& =2 \pi \sum_{n=0}^{\infty} a_{n} \cos (n \pi x),|x| \leq 1 \\
& \int_{-\infty}^{\infty} Q_{+}(\alpha) \cos (\alpha x) d \alpha=0,|x|>1
\end{aligned}
$$

Equations (3) and (4) can be solved using the bilateral asymptotic method [22]. For this purpose, let us approximate the kernel transform by the ratio of two polynomials in the variable $\alpha^{2} \lambda^{2}$

$$
\begin{aligned}
& L(\alpha \lambda) \approx L_{N}(\alpha \lambda)=\frac{P_{1}\left(\alpha^{2} \lambda^{2}\right)}{P_{2}\left(\alpha^{2} \lambda^{2}\right)} \\
& P_{1}(\alpha)=\prod_{i=1}^{N}\left(\alpha+A_{i}^{2}\right), P_{2}(\alpha)=\prod_{i=1}^{N}\left(\alpha+B_{i}^{2}\right)
\end{aligned}
$$

Algorithms for calculation of parameters $A_{i}, B_{i}(i=1, \ldots, N)$ were described earlier [30]

Let us introduce the notation:

$$
p(x)=(2 \pi)^{-1} \int_{-\infty}^{\infty} Q_{-}(\alpha)|\alpha|^{-1} \sin (x \alpha) d \alpha
$$

and rewrite first equation in (3) in the form of differential equation using (5):

$$
P_{1}\left(-\lambda^{2} D^{2}\right) p(x)=P_{2}\left(-\lambda^{2} D^{2}\right)\left(-\sum_{n=1}^{\infty} b_{n} \sin (n \pi x)\right), D=\frac{d}{d x}
$$

Solution of this equation has the form:

$$
\begin{aligned}
& p(x)=\sum_{m=1}^{N}\left(C_{m} \operatorname{ch}\left(A_{m} \lambda^{-1} x\right)+D_{m} \operatorname{sh}\left(A_{m} \lambda^{-1} x\right)\right)- \\
& -\sum_{n=1}^{\infty} b_{n} L_{N}^{-1}(n \pi \lambda) \sin (n \pi x)
\end{aligned}
$$

From the oddity of the function $p(x)$ it follows that $C m=0$ $(m=1, \ldots, N)$. Using (6) and second equation in (3) following dual equation is obtained:

$$
\left\{\begin{array}{l}
\int_{0}^{\infty} Q_{-}(\alpha) \alpha^{-1} \sin (x \alpha) d \alpha=\pi p(x), \quad|x| \leq 1 \\
\int_{0}^{\infty} Q_{-}(\alpha) \sin (x \alpha) d \alpha=0, \quad|x|>1
\end{array}\right.
$$

where $p(x)$ is described in (7). Let us differentiate first equation in (8) with respect to $\mathrm{x}$ and apply an operator $\int_{0}^{t} \frac{d x}{\sqrt{t^{2}-x^{2}}}$, while operator $\int_{t}^{\infty} \frac{d x}{\sqrt{x^{2}-t^{2}}}$ is applied to the second equation

$$
\left\{\begin{array}{l}
\int_{0}^{\infty} Q_{-}(\alpha) J_{0}(t \alpha) d \alpha=2 \int_{0}^{t} \frac{p^{\prime}(x) d x}{\sqrt{t^{2}-x^{2}}}, \quad t \leq 1 \\
\int_{0}^{\infty} Q_{-}(\alpha) J_{0}(t \alpha) d \alpha=0, \quad t>1
\end{array}\right.
$$

Inverting the Hankel transformation in (9) yields

$$
Q_{-}(\alpha)=\pi\left(\sum_{m=1}^{N} \widetilde{D}_{m} \Psi_{-}\left(\alpha, A_{m} \lambda^{-1}\right)-\sum_{n=1}^{\infty} \widetilde{b}_{n} \Psi_{-}(\alpha, i \pi n)\right)
$$

where $\widetilde{D}_{m}=D_{m} A_{m} \lambda^{-1}$ and $\widetilde{b}_{n}=\pi b_{n} n L_{N}^{-1}(n \pi \lambda)$,

$$
\Psi_{ \pm}(x, A)=x \frac{J_{1}(x) I_{0}(A)\{A, x\} \mp\{x, A\} J_{0}(x) I_{1}(A)}{x^{2}-A^{2}}
$$

Let us invert Fourier transformation in (10):

$$
\begin{aligned}
& q_{-}(x)=\frac{1}{2 \pi} \int_{-\infty}^{\infty} Q_{-}(\alpha) e^{-i \alpha x} d \alpha= \\
& \frac{x}{\sqrt{1-x^{2}}}\left[\sum_{n=1}^{\infty} \widetilde{b}_{n} Z_{-}(x, i \pi n)-\sum_{m=1}^{N} \widetilde{D}_{m} Z_{-}\left(x, A_{m} \lambda^{-1}\right)\right]
\end{aligned}
$$

where

$$
\begin{aligned}
& Z_{\{+,-\}}(x, A)=I_{\{1,0\}}(A)+ \\
& +A \frac{\sqrt{1-x^{2}}}{\{-1, x\}} \int_{x}^{1} \frac{t I_{0}(A)\{\mathrm{ch}, \operatorname{sh}\}(A(t-x))-I_{1}(A)\{\mathrm{sh}, \operatorname{ch}\}(A(t-x))}{\sqrt{1-t^{2}}} d t
\end{aligned}
$$

Coefficients $D_{m}(m=1, \ldots, N)$ are calculated from the system of linear algebraic equations below $(n=1, \ldots, N)$ :

$$
\sum_{m=1}^{N} \widetilde{D}_{m} F_{-}\left(A_{m} \lambda^{-1}, B_{n} \lambda^{-1}\right)=\sum_{k=1}^{\infty} \widetilde{b}_{k} F_{-}\left(i \pi k, B_{n} \lambda^{-1}\right)
$$




$$
F_{ \pm}\left(a_{+}, a_{-}\right)=\frac{a_{ \pm} I_{0}\left(a_{+}\right) \mathrm{K}_{1}\left(a_{-}\right)+a_{\mp} I_{1}\left(a_{+}\right) \mathrm{K}_{0}\left(a_{-}\right)}{a_{+}^{2}-a_{-}^{2}}
$$

Solution of dual integral equation (4) was obtained earlier [17, 23, 24]:

$$
\begin{aligned}
& Q_{+}(\alpha)=\widetilde{P} J_{0}(\alpha)-\pi \sum_{m=1}^{N} \widetilde{C}_{m} \Psi_{+}\left(\alpha, \frac{A_{m}}{\lambda}\right)- \\
& -i \pi \sum_{n=1}^{\infty} \widetilde{a}_{n} \Psi_{+}(\alpha, i \pi n) \\
& q_{+}(x)=\left(\frac{\widetilde{P}}{\pi}+\sum_{m=1}^{N} \widetilde{C}_{m} Z_{+}\left(\frac{A_{m}}{\lambda}, x\right)\right) \frac{1}{\sqrt{1-x^{2}}}+ \\
& +i \sum_{n=1}^{\infty} \widetilde{a}_{n} Z_{+}(i \pi n, x) \frac{1}{\sqrt{1-x^{2}}}
\end{aligned}
$$

where $\quad \widetilde{C}_{m}=C_{m} A_{m} \lambda^{-1} \quad$ and $\quad \tilde{a}_{n}=\pi a_{n} n L_{N}^{-1}(n \pi \lambda)$. Coefficients $C_{m}(m=1, \ldots, N)$ are calculated from the system of linear algebraic equations below $(n=1, \ldots, N)$ :

$$
\begin{aligned}
& \sum_{m=1}^{N} \widetilde{C}_{n} F_{+}\left(\frac{A_{m}}{\lambda}, \frac{B_{n}}{\lambda}\right)=\frac{\widetilde{P} \lambda}{\pi B_{n}} \mathrm{~K}_{0}\left(\frac{b_{n}}{\lambda}\right)- \\
& -i \sum_{k=1}^{\infty} a_{k} F_{+}\left(i \pi k, \frac{b_{n}}{\lambda}\right),
\end{aligned}
$$

Thus, solution of the original dual integral equation has the form:

$$
q(x)=q_{+}(x)+q_{-}(x) .
$$

To determine unknown constant $\widetilde{P}$ an additional condition is required. For the indentation problem such condition is equilibrium of the punch: $P=\int_{-1}^{1} q(x) d x$, where $P$ is the dimensionless indentation force.

Expressions (11)-(15) describe the approximated analytical solution of the dual integral equation (1). It was shown [22] that if one approximates the kernel transform in (1) by expression (5) then the obtained solution is asymptotically exact for $\lambda \rightarrow 0$ and $\lambda \rightarrow \infty$. Let us rewrite (1) in the operator form:

$$
\Pi_{N}(p)+\Sigma(p)=f
$$

where $\Pi_{N}(p)=f$ is the approximated equation. Therefore, the discrepancy $\Sigma(p)$ satisfies the following inequalities:

$$
\begin{aligned}
& \max _{x \in(-1,1)}\left|\Sigma(p) \sqrt{1-x^{2}}\right| \leq \lambda M_{0}, \lambda \rightarrow 0\left(\lambda<\lambda_{0}\right), \\
& \max _{x \in(-1,1) \mid}\left|\Sigma(q) \sqrt{1-x^{2}}\right| \leq \frac{M_{\infty}}{\lambda}, \lambda \rightarrow \infty\left(\lambda>\lambda_{\infty}\right),
\end{aligned}
$$

where $\lambda_{0}, \lambda_{\infty}, M_{0}$ and $M_{\infty}$ are some constants independent from $\lambda$. It is obvious that the solution's precision for the intermediate values of $\lambda$ depends on the error of approximation of the kernel transform by expression (5). In the example of torsion problem it was shown [22] that the error of the solution is of the same order of magnitude as the error of the approximation of the kernel transform.

\section{Conclusion}

The results obtained can be used in solution of plane and antiplane contact problems of elasticity theory for solids with coatings (layered or functionally graded). In particular, indentation of an elastic half-plane with a functionally graded coating by a rigid punch with complicated geometry can be reduced to the dual integral equation (1). Full Fourier series in the right-hand side makes it possible to consider a punch with nonsymmetric profile. Using Fourier series one can model additional non-symmetric loading acting in a finite region (region may differ from the contact area). Dual integral equation (1) can be also used in solution of frictional contact problems (for instance, caused by lubricant flow $[26,27,29])$. In this case an iterative process can be organized to calculate contact pressure.

Work was supported by the Russian Foundation for Basic Research (RFBR) (grant numbers 16-07-00958-a, 15-07$05820-a)$ and the President of the Russian Federation (grant number MK-5342.2016.1).

\section{References}

1. L.A. Galin, Kontaktnyye zadachi teorii uprugosti, (Gostekhizdat, Moscow, 1953)

2. N.I. Muskhelishvili, Nekotorye osnovnye zadachi matematicheskoj teorii uprugosti (Nauka, Moscow, 1966)

3. A.E. Giannakopoulos, S Suresh, Int. J. Solids Struct. 34 (1997)

4. M.A. Guler, F. Erdogan, Int. J. Solids Struct., 41 (2004)

5. L.-L. Ke, Y.-S. Wang, Int. J. Solids Struct., 43 (2006)

6. Yu. Tokovyy, C.-C. Ma, J. Appl. Mech., 83 (2016)

7. A.S. Vasiliev, M.V. Swain, S.M. Aizikovich, E.V. Sadyrin, Arch. Appl. Mech., 86 (2016)

8. J. Liu, L.-L. Ke, Y.-S. Wang, J. Yang, F. Alam, Int. J. Mech. Sci. 63 (2012)

9. Yu. Tokovyy, C.-C. Ma, Int. J. Eng. Sci. 92 (2015)

10. L.I. Krenev, Y.V. Tokovyy, S.M. Aizikovich, N.M. Seleznev, S.V. Gorokhov, J. Thermal Stresses, 107 (2016)

11. J. Su, L.-L. Ke, Y.-S. Wang, Int. J. Solids Struct., 90 (2016)

12. A.S. Vasiliev, S.S. Volkov, S.M. Aizikovich, Acta Mech., 227 (2016)

13. G.-Y. Huang, Y.-S. Wang, D. Gross, Eur. J. Mech. Solids., 21 (2002)

14. H. Altenbach, V.A. Eremeyev, Arch. Appl. Mech., 78 (2008)

15. H. Altenbach, V.A. Eremeyev, Computers Mater. Continua, 9 (2009) 
16. S.S. Volkov, A.N. Litvinenko, S.M. Aizikovich, Y.C. Wang, A.S. Vasiliev, Struct. Eng. Mech., 59 (2016)

17. S.M. Aizikovich, B.I. Mitrin, N.M. Seleznev, Y.-C. Wang, S.S. Volkov, Struct. Eng. Mech., 58 (2016)

18. I.I. Vorovich, Iu.A. Ustinov, J. Appl. Math. Mech., 23 (1959)

19. V.M. Aleksandrov, I.I. Vorovich, J. Appl. Math. Mech., 28 (1964)

20. G.Ia Popov, J. Appl. Math. Mech., 33 (1969)

21. A.I. Kalandija, Matematicheskie metody dvumernoj uprugosti (Nauka, Moscow, 1973) [in Russian]

22. S.M. Aizikovich, J. Appl. Math. Mech., 54 (1990)

23. S.M. Aizikovich, S.S. Volkov, B.I. Mitrin, J. Appl. Math. Mech., 81, 6 (2017)

24. A.S. Vasiliev, S.S. Volkov, S.M. Aizikovich, B.I. Mitrin, Z. Angew. Math. Phys., 68 (2017)

25. I.I. Kudish, S.S. Volkov, A.S. Vasiliev, S.M. Aizikovich, J. Tribol., $138 \quad$ (2016) http://dx.doi.org/10.1115/1.4030956

26. I.I. Kudish, S.S. Volkov, A.S. Vasiliev, S.M. Aizikovich, J. Tribol., 138 (2016)

27. I.I. Kudish, S.S. Volkov, A.S. Vasiliev, S.M. Aizikovich, J. Tribol., 139 (2017)

28. I.I. Kudish, S.S. Volkov, A.S. Vasiliev, S.M. Aizikovich, Math. Mech. Solids., (2017)

29. I.I. Kudish, S.S. Volkov, A.S. Vasiliev, S.M. Aizikovich, Math. Mech. Solids., (2017)

30. S.M. Aizikovich, A.S. Vasiliev, J. Appl. Math. Mech. 77 (2013) 